This item was submitted to Loughborough's Research Repository by the author.

Items in Figshare are protected by copyright, with all rights reserved, unless otherwise indicated.

\title{
The validity and reliability of a novel indoor player tracking system for use within wheelchair court sports
}

PLEASE CITE THE PUBLISHED VERSION

http://dx.doi.org/10.1080/02640414.2014.910608

\section{PUBLISHER}

(C) Taylor \& Francis

\section{VERSION}

AM (Accepted Manuscript)

\section{PUBLISHER STATEMENT}

This work is made available according to the conditions of the Creative Commons Attribution-NonCommercialNoDerivatives 4.0 International (CC BY-NC-ND 4.0) licence. Full details of this licence are available at: https://creativecommons.org/licenses/by-nc-nd/4.0/

\section{LICENCE}

CC BY-NC-ND 4.0

\section{REPOSITORY RECORD}

Rhodes, James M., Barry S. Mason, Bertrand Perrat, Martin J. Smith, and Victoria L. Goosey-Tolfrey. 2019. "The Validity and Reliability of a Novel Indoor Player Tracking System for Use Within Wheelchair Court Sports". figshare. https://hdl.handle.net/2134/22651. 
1 Title: The validity and reliability of a novel indoor player tracking system for use within

2 wheelchair court sports

3 Running Title: Radio-frequency player tracking system

4 Keywords: disability sport, field-based testing, accuracy, radio-frequency, performance 5 analysis 


\section{Abstract}

2 The aim of the current study was to investigate the validity and reliability of a radio3 frequency based system for accurately tracking athlete movement within the wheelchair court 4 sports. Four wheelchair specific tests were devised to assess the system during i) static 5 measurements ii) incremental fixed speeds iii) peak speeds, and iv) multi-directional 6 movements. During each test, three sampling frequencies $(4,8$ \& $16 \mathrm{~Hz})$ were compared to a 7 criterion method for distance, mean and peak speeds. Absolute static error remained between $8 \quad 0.19-0.32 \mathrm{~m}$ across the session. Distance values (test ii) showed greatest relative error in $4 \mathrm{~Hz}$ 9 tags (1.3\%), with significantly lower errors seen in higher frequency tags $(<1.0 \%)$. Relative 10 peak speed errors of $<2.0 \%$ (test iii) were revealed across all sampling frequencies in relation 11 to the criterion $\left(4.00 \pm 0.09 \mathrm{~m} \cdot \mathrm{s}^{-1}\right)$. Results showed 8 and $16 \mathrm{~Hz}$ sampling frequencies 12 displayed the closest to criterion values, whilst intra-tag reliability never exceeded $2.0 \%$ 13 coefficient of variation (\% CV) during peak speed detection. Minimal relative distance errors 14 (<0.2\%) were also seen across sampling frequencies (test iv). To conclude, the indoor 15 tracking system is deemed an acceptable tool for tracking wheelchair court match-play using 16 a tag frequency of 8 or $16 \mathrm{~Hz}$. 


\section{Introduction}

2 Understanding the movement demands placed upon an athlete during competition is a 3 fundamental requirement for the prescription of specific, individualised training programmes. 4 Player tracking has been extensively used within able-bodied (AB) team sports to explore 5 movement demands, with basic notational techniques employed since the mid-1970's (Reilly 6 \& Thomas, 1976; Sanderson \& Way, 1977). Advances in technology introduced more 7 objective methods of player tracking, such as manual (O’Donoghue, 2002; Bloomfield, 8 Polman, \& O’Donoghue, 2004) and automatic video tracking techniques (Figueroa, Leite, \& 9 Barros, 2006; Barros et al., 2007). Currently, the use of Global Positioning Systems (GPS) 10 has emerged as the most practical method for of player tracking multiple player movements to obtain a real time analysis of key performance variables (e.g. distance covered and speed profiles) 2013). This technology was first utilised to understand more about sports performance in the late 1990's (Schutz \& Chambaz, 1997) and has been increasingly used by sport scientists in team sports environments since (Cummins, Orr, O’Connor, \& West, 2013).

The validity of GPS during high intensity, intermittent sportsing environments-has been comprehensively examined (MacLeod, Morris, Nevill, \& Sunderland, 2009; Duffield, Reid, Baker, \& Spratford, 2010; Coutts \& Duffield, 2010; Portas, Harley, Barnes, \& Rush 2010; Johnston et al., 2012). These iInvestigations suggest that GPS accurately tracks players during low-speed $\left(<1.8 \mathrm{~m} \cdot \mathrm{s}^{-1}\right)$ movements (Portas, Rush, Barnes, \& Batterham, 2007) $)_{2}=$ However, during high-speed movements $\left(>4 \mathrm{~m} \cdot \mathrm{s}^{-1}\right)$ greater-with distance and speed errors (5-20\%) increasing exponentially during high-speed $\left(>4 \mathrm{~m} \cdot \mathrm{s}^{-1}\right)$ movements reported (Petersen, Pyne, Portus, \& Dawson, 2009;-Duffield et al., 2010;-Gray, Jenkins, Andrews, Taaffe, \& Glover, 2010; Johnston et al., 2012). Recent studies have also revealed that the validity and reliability of GPS improves when higher sampling frequencies $(10 \mathrm{~Hz})$ are used, which is likely to have contributed contributing towards the magnitude of these aforementioned errors (Petersen et al., 2009; Jennings, Cormack, Coutts, Boyd, \& Aughey, 2010; Castellano et al., 2011; Varley, Fairweather, \& Aughey, 2012).

A major limitation with GPS is its reliance on satellite signals, restricting its use to an outdoor environment only (Larsson, 2003). As a result, indoor team sports such as wheelchair basketball and wheelchair rugby (known collectively as the wheelchair court sports), cannot utilise GPS. Consequently, image-based processing techniques (Sarro, Misuta, Burkett, Malone, \& Barros, 2010) and wheel mounted magnetic reed-switch devices (Sporner et al., 
1 2009; Sindall et al., 2013a) have been employed in an attempt to determine the demands of

2 the wheelchair court sports. Unfortunately, there are limitations associated with both these

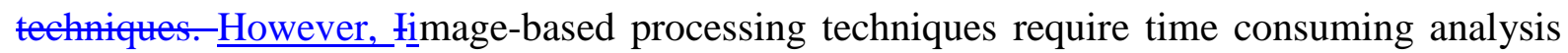

4 to be performed post event (Barris \& Button, 2008), which introduces accuracy and reliability

5 issues due to a heavy reliance on manual digitisation (Lames \& Siegle, 2011) along with a

6 delay in feedback time to coaches. Subsequently, data collection is often restricted to small

7 sample sizes, affecting the power of such investigations. Substantial errors in measurement

8 reliability (19.9\% coefficient of variation [\% CV]) have also been reported within magnetic

9 reed-switch devices at speeds in excess of $2.5 \mathrm{~m} \cdot \mathrm{s}^{-1}$ (Sindall et al., 2013b). Such speeds are frequently exceeded by elite wheelchair athletes (Goosey-Tolfrey \& Moss, 2005; Mason et al., 2009; 2012), which questions the suitability of existing reed-switch devices for use within elite wheelchair court sport applications.

Radio-frequency tracking systems have emerged, which gather similar data to GPS, with both the Local Position Measurement (LPM) system (Frencken, Lemmink, \& Delleman, 2010; Ogris et al., 2012) and the Wireless Ad-hoc System for Positioning (WASP) (Hedley et al., 2010; Sathyan, Shuttleworth, Hedley, \& Davids, 2011) currently available. These systems rely on distance measurements between known fixed base stations and mobile tags worn by the athlete (Leser, Baca, \& Ogris, 2011). A key advantage of these-radio-frequency systems is that they can function indoors (Sathyan, Humphrey, \& Hedley, 2011). Unfortunately, these systems are still in their relative infancy, particularly for sporting applications and as a result little is known about their validity and reliability. Initial validation of the LPM system when sampling at $45 \mathrm{~Hz}$, highlighted the typical error of the estimate increased (1.8-3.9\% CV) at higher movement speeds (Frencken et al., 2010). In support of this, Ogris et al. (2012) confirmed error values increased (10\% error) during high speed movements, yet the LPM system provided valid speed estimations at low speeds $\left(<6 \mathrm{~km} \cdot \mathrm{h}^{-1}\right)$. More recently, validation of the WASP system when sampling at $10 \mathrm{~Hz}$, revealed an overestimation (2.7\%) in distance travelled during dynamic testing (Sathyan et al., 2012). Unfortunately, the analysis was confined to a basic linear and non-linear drill at self-regulated speeds (not defined), which may not adequately reflect athlete movements seen during match-play.

A new, radio frequency-based indoor tracking system (ITS) has recently been developed, which utilises ultra-wideband (UWB) signals to communicate with compact tags worn by athletes, providing real-time analysis on movement parameters. The additional benefit of the ITS is the incorporation of smaller, lightweight tags (size $=40 \times 40 \times 10 \mathrm{~mm}$; mass $=25 \mathrm{~g})$, opposed to the larger tags used with the WASP $(90 \times 50 \times 25 \mathrm{~mm}$; mass $=50 \mathrm{~g})$ 
1 and-LPM (92 × 57 × 15 mm; mass = 60 g) and WASP $(90 \times 50 \times 25$ mm; mass = 50 g) systems. Subsequently, the system-ITS may be a more practical solution since minimal

3 disruption would be imposed on athletes during competition and training environments.

4 Therefore the aims of the current study were: (1) to investigate the validity and reliability of 5 the ITS during movements and speeds specific to the wheelchair court sports and (2) to 6 determine the effect of different sampling frequencies on the system's measurement accuracy.

\section{Methods}

9 Participants

10 Two physically active, able-bodied males (age: $30.0 \pm 2.0$ years, mass: $82.5 \pm 9.2 \mathrm{~kg}$, height:

$111.81 \pm 0.04 \mathrm{~m}$ ) with extensive experience of wheelchair propulsion volunteered to participate 12 in the current investigation. The study was approved by the University's local ethical 13 advisory committee, with informed consent gained prior to participation.

\section{Equipment}

15 The ITS (Ubisense, Series 700 IP, Cambridge, UK) is a wired radio-frequency based real16 time location system. The system has an overall bandwidth of $137 \mathrm{~Hz}$ and is comprised of six 17 sensors that communicate with compact tags. The sensors detect UWB signals from the tags, 18 measuring both the angle-of-arrival and the time-difference-of-arrival to generate an accurate 19 tag location. This provides raw data on the positional coordinates of a tag in three dimensions. 20 Raw data is then filtered using a 3-pass sliding-average filter with a window width 21 proportional to the tag frequency.

The validity and reliability of the ITS was assessed during one session using four 23 separate tests i) static measurements; ii) incremental fixed speeds; iii) peak speeds; iv) multi24 directional movements. Movement parameters detailed by the ITS were derived using 25 software developed specifically for wheelchair court sports at the University of Nottingham. 26 All dynamic tests (ii, iii \& iv) were performed in a rugby wheelchair (Melrose Wheelchairs, 27 New Zealand: mass $=12.7 \mathrm{~kg}$; wheel size $=0.591 \mathrm{~m}$; tyre pressure $=120 \mathrm{psi}$; camber $=18^{\circ}$ ). 28 The criterion measurement for distance (tests ii \& iv) was provided by a laser total station (Leica TS-30, Leica Geosystems, UK), more commonly used within a professional surveying environment. The Leica system utilises high quality angle and distance measurements with automatic target tracking to produce accurate coordinates $(\sim 0.004 \mathrm{~m})$ about the point of 
1 interest (Bayoud, 2006). The total station was positioned on a balcony overlooking the entire 2 court, ensuring a consistent, unobstructed view throughout each test. Wireless timing gates 3 (Brower Timing Systems, Draper, UT) were used to record the mean speed (tests ii and iv), 4 whilst a wireless inertial sensor (Ellul, Lo, \& Yang, 2011), attached to the right axle of the 5 wheelchair provided the criterion measurement for peak speed (test iii). In brief, the inertial sensor is a small, lightweight device (size $=20 \times 30 \times 17 \mathrm{~mm}$; mass $=10 \mathrm{~g}$ ) that transmits data wirelessly at a sampling frequency of approximately $50 \mathrm{~Hz}$. This device has previously been validated during linear wheelchair propulsion (Mason, Rhodes, \& Goosey-Tolfrey, 2013a), reporting speed errors $<0.9 \% \mathrm{CV}$ observed across a range of speeds up to $6 \mathrm{~m} \cdot \mathrm{s}^{-1}$.

Procedures

11 The ITS was set up in an indoor sports hall equipped with wooden sprung flooring to replicate the playing surface used during wheelchair basketball and wheelchair rugby. The six sensors were located around the perimeter of a regulation size wheelchair basketball and wheelchair rugby court ( $28 \times 15 \mathrm{~m}$ ). The sensors were positioned at each of the four corners of the court, with two additional sensors positioned at the half-way line. Each sensor was mounted on an extendable tripod, elevated approximately $4 \mathrm{~m}$ high. The orientation of each sensor was configured so that the pitch was $40^{\circ}$ from the horizontal and the rotation about the perpendicular line from the sensor face was fixed at $0^{\circ}$, maximising court coverage. Prior to data collection the system was calibrated using two reference points of known coordinates, which were calculated by a laser distance measurer (PLR 50, Bosch, Germany). This enabled precise sensor locations to be determined. A static tag placed in another known location was then used to calibrate the system. This procedure takes multiple measurements from the static tag using its known $\mathrm{x}, \mathrm{y}$ and $\mathrm{z}$ coordinates to determine the orientation and offset off each sensor (Mandeljc, Perš, Kristan, \& Kovačič, 2012). During all dynamic tests (tests ii, iii \& iv) nine tags were monitored, with three tags sampling at a low $(4 \mathrm{~Hz})$, medium $(8 \mathrm{~Hz})$, and high $(16 \mathrm{~Hz})$ frequency, which were secured to the wheelchair as demonstrated in Figure 1.

The accuracy of a motionless tag was assessed by individually placing three tags of different sampling frequency (low, medium and high) in each of the four corners of the court (where known coordinates exist). Based on previous protocols (Frencken et al., 2010; Sathyan et al., 
1 2012) data was collected from each tag for 20 seconds. This assessment was performed at the

2 beginning of the session (pre) and then repeated 4 hours later at the end of the session (post)

3 to determine whether the system was prone to drift over time.

4

5

6 7

\section{ii) Incremental fixed speeds}

The accuracy of the system for detecting distance measurements was assessed over increasing fixed speeds using a 'figure of eight' course (Figure 2). One participant completed five laps of the course at three fixed sub-maximal speeds $\left(4 \mathrm{~km} \cdot \mathrm{h}^{-1}, 6 \mathrm{~km} \cdot \mathrm{h}^{-1}\right.$, and $\left.8 \mathrm{~km} \cdot \mathrm{h}^{-1}\right)$, with five trials conducted at each speed. The speeds selected are commonly used within previous submaximal wheelchair propulsion literature (Vanlandewijck, Spaepen \& Lysens, 1994; Mason, Lenton, Leicht, \& Goosey-Tolfrey, 2013b). This range also covers the speeds typically averaged during wheelchair court sports match-play (Sporner et al., 2009; Sarro et al., 2010). The speeds were averaged throughout each trial through using a Raleigh SP-20 speedometer (Raleigh Ltd, Nottingham, UK). The display monitor was secured to the participant's knee, providing instantaneous feedback about their average speed. The participant was instructed to maintain these speeds, on average, throughout each trial.

\section{****INSERT FIGURE 2 HERE****}

\section{iii) Peak speeds}

To assess the accuracy of the system for the detection of peak speeds, a $20 \mathrm{~m}$ linear wheelchair sprint was performed. One participant completed all ten trials from a standstill. After each maximal effort, sufficient recovery time was permitted before each subsequent sprint.

\section{iv) Multi-directional movements}

In order to determine the accuracy of a player tracking system, the experimental design has to satisfy the demands of the activity to which the system will be exposed (Siegle et al., 2013). A multi-directional drill was performed aimed to replicate the frequency and intensity of movements performed during wheelchair court sports match-play. Two participants performed $4 \times$ 8-min trials in an alternate order to avoid the possibility of fatigue affecting the quality of the trials, resulting in a total of $8 \times 8$-min trials. The participants were instructed to incorporate numerous changes in speed and direction to replicate the acceleration, agility and sprinting manoeuvres deemed vital to wheelchair court sport athletes (Vanlandewijck, 
1 Theisen, \& Daly, 2001). The total distances covered and mean speeds were collected during

2 each trial.

3 Statistical analysis

4 Data analysis was performed using the Statistical Package for the Social Sciences (SPSS 5 version 19, Chicago, IL). Normality and homogeneity of variance were confirmed by 6 Shapiro-Wilk and Levene’s tests, respectively.

Criterion validity of the performance variables measured by the ITS were analysed using 95\% limits of agreement (LOA), displaying the systematic bias \pm random error demonstrated for each variable (Bland \& Altman, 1986). During test ii, validity was also compared to criterion measures using the typical error of the estimate (TEE) and expressed in raw units ( $\pm 95 \%$ confidence limits). A one-way repeated measures analysis of variance (ANOVA) was used to examine the mean differences in performance variables within and between each of the three different sampling frequencies compared to criterion measures across all tests. Statistical significance was accepted when $P<0.05$. Effect sizes (ES) were calculated to determine the meaningfulness of any differences, whereby $\mathrm{ES}<0.3$ reflected a small effect (Cohen, 1992), with 95\% confidence intervals for differences (95\% CI) also presented-analysed. Intra-tag reliability was reported as a coefficient of variation (\% CV) between the tags for each specific test.

\section{Results}

\section{(i) Static measurements}

21 The mean absolute error during pre-session measurements did not significantly differ between low $(0.24 \pm 0.27 \mathrm{~m})$, medium $(0.26 \pm 0.25 \mathrm{~m})$ and high $(0.32 \pm 0.25 \mathrm{~m})$ frequency tags $(P \geq 0.72$; ES $\leq 0.1)$, as demonstrated in Figure 3 . No significant differences in post session values were revealed between low $(0.26 \pm 0.24 \mathrm{~m})$, medium $(0.26 \pm 0.24 \mathrm{~m})$ or high frequency $(0.19 \pm 0.20 \mathrm{~m})$ tags $(P \geq 0.92 ; \mathrm{ES} \leq 0.2)$. No significant differences between pre and post session measurements were found at any sampling frequency ( $P \geq 0.15$; ES $\leq 0.2)$.

27 Intra-tag reliability results revealed that sampling frequency had no effect on reliability with a 1.0\% CV demonstrated across all frequencies during pre and post session measurements. 
1 The TEE for distance revealed that minimal errors existed during high and medium fixed 2 speeds (0.98-1.09 m), however values increased during low fixed speed (1.85-2.11 $\mathrm{m})$ as

3 displayed in Table 1. A significant difference existed between criterion measures and low ( $P$

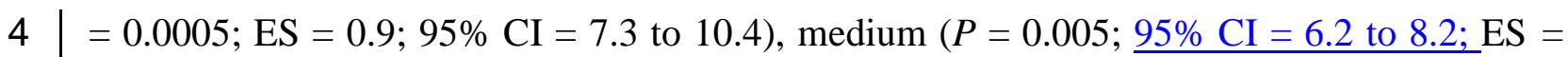
$0.8 ; 95 \% \mathrm{CI}=6.2$ to 8.2$)$, and high $(P=0.005 ; 95 \% \mathrm{CI}=4.5$ to $6.6 ; \mathrm{ES}=0.8 ; 95 \% \mathrm{CI}=4.5$ to 6.6) sampling frequencies during low fixed speeds. However, no significant differences were observed during the medium and high fixed speeds ( $P \geq 0.12$; ES $\leq 0.7)$. Typical error

8 of the estimate values for mean speed demonstrate the ITS to be consistent $\left(0.01 \mathrm{~m} \cdot \mathrm{s}^{-1}\right)$ across all sampling frequencies at each fixed speed. Although low frequency tags displayed the greatest absolute differences to criterion values (Table 1), no statistically significant difference was observed between sampling frequencies for mean speed ( $P \geq 0.15$; ES $\leq 0.4$ ). Intra-tag reliability results indicated that the error range across fixed speeds to be greatest within low frequency tags $(0.1-0.6 \% \mathrm{CV})$. This error range decreased at both medium (0.2$0.4 \% \mathrm{CV})$ and high (0.2-0.3\% CV) sampling frequencies.

(iii) Peak speeds

Mean criterion values were found to be $4.00 \pm 0.09 \mathrm{~m} \cdot \mathrm{s}^{-1}$ during maximal sprint trials. In comparison, mean tag values for each sampling frequency were $4.07 \pm 0.14 \mathrm{~m} \cdot \mathrm{s}^{-1}$ (low), 4.05 $\pm 0.15 \mathrm{~m} \cdot \mathrm{s}^{-1}$ (medium), and $4.00 \pm 0.12 \mathrm{~m} \cdot \mathrm{s}^{-1}$ (high). A significant difference was revealed

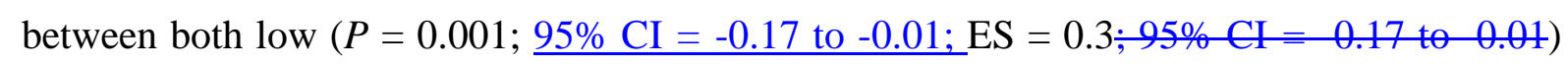

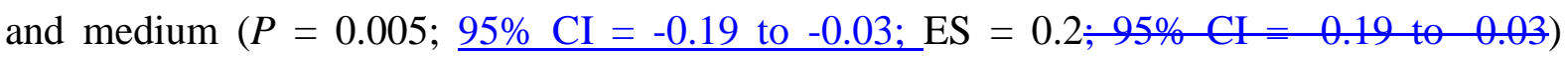
sampling frequencies in relation to the criterion measure, with positive systematic bias \pm random errors of $0.08 \pm 0.17 \mathrm{~m} \cdot \mathrm{s}^{-1}$ and $0.05 \pm 0.10 \mathrm{~m} \cdot \mathrm{s}^{-1}$ respectively (Figure 4). Intra-tag reliability was greater within low frequency tags $(2.7 \% \mathrm{CV})$, and improved as sampling frequency increased $($ medium $=2.0 \% \mathrm{CV}$; high $=1.6 \% \mathrm{CV}$ ).

\section{****INSERT FIGURE 4 HERE****}

\section{(iv) Multi-directional movements}

Mean criterion distance measurements were $999 \pm 65 \mathrm{~m}$ during the multi-directional test. In comparison, mean distance values for each sampling frequency were $997 \pm 63 \mathrm{~m}$ (low), $999 \pm$ $63 \mathrm{~m}$ (medium) and $998 \pm 62 \mathrm{~m}$ (high). Criterion values for mean speed were $2.08 \pm 0.14$ $\mathrm{m} \cdot \mathrm{s}^{-1}$. Alternatively, ITS mean speed values showed $2.08 \pm 0.13 \mathrm{~m} \cdot \mathrm{s}^{-1}$ (low), $2.08 \pm 0.13$ 
$1 \mathrm{~m} \cdot \mathrm{s}^{-1}$ (medium), and $2.07 \pm 0.13 \mathrm{~m} \cdot \mathrm{s}^{-1}$ (high). Systematic bias and random error values for

2 distance and mean speed during the 8-minute multi-directional test are illustrated in Figure 5.

3 Distance results show the low and medium frequency tags to demonstrate similar systematic

4 bias \pm random error $(5 \pm 10 \mathrm{~m})$, which were improved in the high frequency tags $(3 \pm 6 \mathrm{~m})$.

5 Yet, no significant difference was observed between any tag frequency and the criterion

6 measure for distance covered $(P \geq 0.54$; ES $\leq 0.1)$. Systematic bias \pm random error results for

7 mean speed remained consistent across all sampling frequencies $\left(0.01 \pm 0.02 \mathrm{~m} \cdot \mathrm{s}^{-1}\right)$. Again,

8 no significant differences were identified between all sampling frequencies and the criterion

9 measure for mean speed ( $P \geq 0.71$; ES $\leq 0.1)$. Intra-tag reliability results revealed $0.5 \% \mathrm{CV}$

10 for both distance and mean speed in low and medium frequency. High frequency tags

11 revealed values of $0.2 \% \mathrm{CV}$ and $0.4 \% \mathrm{CV}$ for distance and mean speed respectively.

13 Discussion

14 The aim of the current study was to investigate the validity and reliability of a radio15 frequency based system for accurately tracking wheelchair athletes during their expected 16 movements of on-court match-play. The results confirmed that the ITS was a suitable system 17 for quantifying both static and dynamic measurements specific to wheelchair court sports. It 18 was also revealed that sampling frequency influenced validity, particularly at peak speeds, which has implications on optimal tag frequency selection for wheelchair court sports applications.

\section{Static measurements}

22 The ITS elicited static errors ranging between 0.19-0.32 $\mathrm{m}$ and were not found to be 23 influenced by tag sampling frequency. These values are higher than those previously reported 24 for the LPM $(0.02 \mathrm{~m})$ and WASP $(0.12-0.18 \mathrm{~m})$ radio frequency systems (Frencken et al., 25 2010; Sathyan et al., 2011). Despite this, the current investigation repeated the static 26 measurements at the end of the testing session and importantly revealed that error did not 27 significantly drift over a 4 hour time period. From a practical perspective, this demonstrates 28 that the ITS is capable of working effectively for the duration of wheelchair basketball ( $\sim 90$ 29 minutes) and wheelchair rugby ( 60 minutes) match-play. In addition, the ITS can also be 30 used during prolonged periods, such as multiple tournament games (3-4 matches per day) and training camps, without the concern of measurement drift. 
2 Under controlled testing at incremental fixed speeds (test ii) the ITS demonstrated extremely

3 low errors for the assessment of distance covered. As expected, these errors were influenced

4 by movement speed. However, it was observed that the magnitude of error was reduced at the

5 higher speed, which contradicts the patterns observed by previous GPS (Peterson, Pyne,

6 Portus, \& Dawson, 2009; Gray, Jenkins, Andrews, Taaffe, \& Glover, 2010), radio-frequency

7 (Frencken et al., 2010; Ogris et al., 2012), and magnetic reed-switch device literature (Sindall

8 et al., 2013b). These differences may be attributed to the filtering process used by the ITS, as

9 if a small error exists in a specific court location, the filtering process used may exacerbate

10 the error at low speeds, where more data points are collected for a given area. Since low point

11 (< 1.5) wheelchair rugby players exhibit mean speeds of $0.78-1.12 \mathrm{~m} \cdot \mathrm{s}^{-1}$ during match-play

12 (Sporner et al., 2009; Sarro et al., 2010), it is imperative that the system works effectively at 13 these lower speeds. However, despite the fact the distance error was greater at low speeds it 14 must be reinforced, that these errors were still extremely small (1.96-2.11 m TEE) and are 15 therefore deemed acceptable for the current application.

The influence of sampling frequency can be seen during this drill, with low frequency 17 tags demonstrating the greatest relative distance error values (1.3\%), with significantly lower relative errors seen in medium (1.0\%) and high frequency tags (0.8\%). In agreement with this, mean speed results also revealed low frequency tags to display the greatest relative differences during fixed speed testing (1.4\%), with significantly lower relative errors seen in medium $(0.7 \%)$ and high frequency tags (0.5\%). Nevertheless, TEE values for mean speed were minimal (0.01) and remained consistent across all fixed speeds regardless of sampling frequency.

24 Peak speeds

The current study revealed that during maximal sprinting, the ITS displayed relative errors < $2.0 \%$ in peak speeds. This compares favourably to the greater relative error of approximately 20\% for GPS (Duffield et al., 2010), 10\% in radio frequency (Ogris et al., 2012) and 10\% for magnetic reed-switch devices (Sindall et al., 2013b). Previous research has discussed the importance of accurately quantifying high intensity movements to facilitate the design of athlete training programmes (Dwyer \& Gabbett, 2012). Recent studies have implemented the use of speed zones relative to an individual's peak speed in order to monitor performance and prescribe training programmes (Venter et al., 2011; Cahill et al., 2013). In order for this 
1 approach to be effective, the system must be capable of accurately quantifying peak speeds,

2 which the present results have confirmed.

It was also clear that tag frequency played a critical role in accurately identifying peak speeds. Higher tag frequencies (8 and $16 \mathrm{~Hz}$ ) demonstrated a reduction in random error (< $\left.0.10 \mathrm{~m} \cdot \mathrm{s}^{-1}\right)$ compared to low frequency tags $\left(0.17 \mathrm{~m} \cdot \mathrm{s}^{-1}\right)$. Given the peak speed values obtainable by wheelchair basketball $\left(4.45-4.53 \mathrm{~m} \cdot \mathrm{s}^{-1}\right)$ and wheelchair rugby $\left(3.56-3.69 \mathrm{~m} \cdot \mathrm{s}^{-1}\right)$ players during maximal sprinting (Mason et al., 2009; 2012), coupled with the frequency with which high-intensity movements are likely to be performed (Vanlandewijck et al., 2001) low sampling frequency tags were therefore not deemed suitable for the current application.

\section{Multi-directional movements}

An advantage of the current investigation was the inclusion of a test which assessed the ITS during the type and intensity of movements that the system was intended to be used for (Siegle et al., 2013) i.e. wheelchair court sports. Distance errors revealed when performing multi-directional movements were very low, with absolute errors $<2 \mathrm{~m}$ across sampling frequencies, resulting in relative errors $<0.2 \%$. The magnitude of error for the ITS was much smaller than the relative distance errors of 5.8\% associated with GPS (Duffield et al., 2010), 4.8\% with video tracking techniques (Edgecomb \& Norton, 2006) and 1.6-2.7\% found in radio-frequency systems (Frencken et al., 2010; Ogris et al., 2012) during sport specific movements.

During this drill, minimal absolute differences in distance (1-2 m) were seen when comparing sampling frequencies. Additionally, similar findings were observed in the mean speed results, with relative errors consistent $(<0.3 \%)$ irrespective of sampling frequency. Clearly, the influence of sampling frequency seems to be more prevalent during the incremental fixed speed test (test ii) than the current test. In line with previous research, this suggests that the validity of distance measures improves with longer duration activities (Jennings et al., 2011; Cummins et al., 2013). Accordingly, the selection of sampling frequency for the assessment of distance and mean speed may be less important during wheelchair court sport match-play. Despite this, optimal sampling frequency must be considered for an accurate detection of peak speeds during this application.

Determining the optimal tag sampling frequency depends on both the overall bandwidth of the system and the nature of the sport. The likelihood of competition testing 
1 during wheelchair court sports consists of monitoring 8-10 players at a given time, yet given

2 the overall bandwidth of the system $(137 \mathrm{~Hz})$, high frequency tags $(16 \mathrm{~Hz})$ would not be

3 feasible for all players. Hence, low or medium sampling frequency tags would be required.

4 Yet, by varying the sampling frequencies within the present study, the differences observed

5 have established that adopting a methodology that uses high $(16 \mathrm{~Hz})$ or medium $(8 \mathrm{~Hz})$

6 sampling frequency would be most acceptable for wheelchair court sports match-play.

$7 \quad$ Limitations and future recommendations

8 A limitation of the current study was the use of linear $20 \mathrm{~m}$ sprints to assess high intensity 9 activities, since these movements are often multidirectional and interspersed in between 10 lower intensity movements in wheelchair sports (Vanlandewijck et al., 2001). However 11 owing to limitations with the availability of alternative equipment available to act as a valid 12 and reliable criterion measure this was not possible. Previous research has also discussed the 13 importance of quantifying activity into relative and arbitrary speed thresholds to facilitate 14 training programme development (Dwyer \& Gabbett, 2012; Cahill et al., 2013). It could be 15 argued that an assessment of these parameters may have been beneficial in the context of the current investigation. However, given the favourable performance in the detection of peak speeds, it is anticipated that the ITS should adequately determine these parameters. Given the validity and reliability of the ITS in a wheelchair court sport setting, future investigations are recommended to utilise the system to quantify the demands of these sports. This would build on the limited existing knowledge within the wheelchair court sports (Sarro et al., 2010) and facilitate exercise prescription specific to the needs of individual athletes. Ddespite the current focus on wheelchair courts sports, it is possible that the ITS can also be used successfully within other indoor team-sports. However, it is highly recommended that a

24 validation protocol specific to these sports are employed first.

\section{Conclusion}

26 The results of the present study revealed that a novel radio frequency ITS provided an 27 accurate and reliable quantification of the movement parameters specific to the wheelchair 28 court sports. Given the greater degree of accuracy for detecting peak speeds, a high sampling frequency ( $\geq 8 \mathrm{~Hz}$ ) was recommended for use within wheelchair court sports. 
2

Barris, S., \& Button, C. (2008). A review of vision-based motion analysis in sport. Sports Medicine, 38, 1025-1043.

Barros, R. M., Misuta, M. S., Menezes, R. P., Figueroa, P. J., Moura, F. A., Cunha, S. A., et al. (2007). Analysis of the distances covered by first division Brazilian soccer players obtained with an automatic tracking method. Journal of Sports Science and Medicine, 6, 233-242.

Bayoud, F. (2006). Leica's PinPoint EDM Technology with Modified Signal Processing and Novel Optomechanical Features. In: Proceedings of XXIII FIG Congress, Munich, 2006, 1-16.

Bloomfield, J., Polman, R., \& O'Donoghue, P. (2004). The 'Bloomfield Movement Classification': motion analysis of individual players in dynamic movement sports. International Journal of Performance Analysis in Sport, 4, 20-31.

Cahill, N., Lamb, K., Worsfold, P., Headey, R., \& Murray, S. (2013) The movement characteristics of English Premiership rugby union players. Journal of Sport Sciences, 31, 229-237.

Castellano, J., Casamichana, D., Calleja-González, J., San Román, J., \& Ostojic, S. M. (2011). Reliability and accuracy of $10 \mathrm{~Hz}$ GPS devices for short-distance exercise. Journal of Sports Science and Medicine, 10, 233-234.

Cohen, J (1992) A power primer. Psychological Bulletin, 112, 155-159.

Coutts, A. J., \& Duffield, R. (2010). Validity and reliability of GPS devices for measuring movement demands of team sports. Journal of Science and Medicine in Sport, 13, 133135.

Cummins, C., Orr, R., O’Connor, H., \& West, C. (2013). Global positioning systems (GPS) and microtechnology sensors in team sports: a systematic review. Sports Medicine, 43, 1025-1042.

Duffield, R., Reid, M., Baker, J., \& Spratford, W. (2010). Accuracy and reliability of GPS devices for measurement of movement patterns in confined spaces for court-based sports. Journal of Science and Medicine in Sport, 13, 523-525.

Dwyer, D., \& Gabbett, T. (2012) Global positioning system data analysis: velocity ranges and a new definition of sprinting for field sport athletes. Journal of Strength and Conditioning Research. 26, 818-824.

Edgecomb, S. J., \& Norton, K. I. (2006). Comparison of global positioning and computerbased tracking systems for measuring player movement distance during Australian football. Journal of Science and Medicine in Sport, 9, 25-32. 
Ellul, J., Lo, B., \& Yang, G. Z. (2011). The BSNOS platform: a body sensor networks targeted operating system and toolset. In proceedings of SENSORCOMM 2011, The Fifth International Conference on Sensor Technologies and Applications, 381-386.

Figueroa, P. J., Leite, N. J., \& Barros, R. M. (2006). Tracking soccer players aiming their kinematical motion analysis. Computer Vision and Image Understanding, 101, 122-135.

Frencken, W. G., Lemmink, K. A., \& Delleman, N. J. (2010). Soccer-specific accuracy and validity of the local position measurement (LPM) system. Journal of Science and Medicine in Sport, 13, 641-645.

Goosey-Tolfrey, V. L., \& Moss, A. D. (2005). Wheelchair velocity of tennis players during propulsion with and without the use of racquets. Adapted Physical Activity Quarterly, 22, 291-301.

Gray, A. J., Jenkins, D., Andrews, M. H., Taaffe, D. R., \& Glover, M. L. (2010). Validity and reliability of GPS for measuring distance travelled in field-based team sports. Journal of Sports Sciences, 28, 1319-1325.

Hedley, M., Mackintosh, C., Shuttleworth, R., Humphrey, D., Sathyan, T., \& Ho, P. (2010). Wireless tracking system for sports training indoors and outdoors. Procedia Engineering, 2, 2999-3004.

Jennings, D., Cormack, S., Coutts, A. J., Boyd, L. J., \& Aughey, R. J. (2010). Variability of GPS units for measuring distance in team sport movements. International Journal of Sports Physiology and Performance, 5, 565-569.

Johnston, R. J., Watsford, M. L., Pine, M. J., Spurrs, R. W., Murphy, A. J., \& Pruyn, E. C. (2012). The validity and reliability of 5-hz global positioning system units to measure team sport movement demands. The Journal of Strength \& Conditioning Research, 26, 758-765.

Lames, M., \& Siegle, M. (2011). Positional data in game sports - Validation and practical impact. In Y. Jiang \& H. Zhang (Eds.), Proceedings of the VIII International Symposium of Computer Science in Sport (pp. 5-9). Shanghai, China: World Academic Union.

Larsson, P. (2003). Global positioning system and sport-specific testing. Sports Medicine, 33, 1093-1101.

Leser, R., Baca, A., \& Ogris, G. (2011). Local positioning systems in (game) sports. Sensors, 11, 9778-9797.

MacLeod, H., Morris, J., Nevill, A., \& Sunderland, C. (2009). The validity of a nondifferential global positioning system for assessing player movement patterns in field hockey. Journal of Sports Sciences, 27, 121-128.

Mandeljc, R., Kovačič, S., Kristan, M., \& Perš, J. (2012). Tracking by identification using computer vision and radio. Sensors, 13, 241-273. 
Mason, B. S., van der Woude, L. H., \& Goosey-Tolfrey, V. L. (2009). Influence of glove type on mobility performance for wheelchair rugby players. American Journal of Physical Medicine \& Rehabilitation, 88, 559-570.

Mason, B., van der Woude, L., Tolfrey, K., \& Goosey-Tolfrey, V. (2012). The effects of rearwheel camber on maximal effort mobility performance in wheelchair athletes. International Journal of Sports Medicine, 33, 199-204.

Mason, B., Rhodes, J., Goosey-Tolfrey, V. (2013a) Validity and reliability of an inertial sensor for wheelchair court sports performance. Journal of Applied Biomechanics (Epub ahead of print).

Mason, B., Lenton, J., Leicht, C., \& Goosey-Tolfrey, V. (2013b). A physiological and biomechanical comparison of over-ground, treadmill and ergometer wheelchair propulsion. Journal of Sports Sciences, DOI: 10.1080/02640414.2013.807350.

O’Donoghue, P. G. (2002). Time-motion analysis of work-rate in English FA Premier League soccer. International Journal of Performance Analysis in Sport, 2, 36-43.

Ogris, G., Leser, R., Horsak, B., Kornfeind, P., Heller, M., \& Baca, A. (2012). Accuracy of the LPM tracking system considering dynamic position changes. Journal of Sports Sciences, 30, 1503-1511.

Petersen, C., Pyne, D., Portus, M., \& Dawson, B. (2009). Validity and reliability of GPS units to monitor cricket-specific movement patterns. International Journal of Sports Physiology and Performance, 4, 381-393.

Portas, M. D., Rush, C. J., Barnes, C. A., \& Batterham, A. M. (2007). Method comparison of linear distance and velocity measurements with global positioning satellite (GPS) and the timing gate techniques. Journal of Sport Science and Medicine, 6 (suppl.10), 7-8.

Portas, M. D., Harley, J. A., Barnes, C. A., \& Rush, C. J. (2010). The validity and reliability of $1 \mathrm{hz}$ and $5 \mathrm{hz}$ global positioning systems for linear, multidirectional, and soceer specific activities. International Journal of Sports Physiology and Performance, 5, 448 458.

Reilly, T., \& Thomas, V. (1976). A motion analysis of work-rate in different positional roles in professional football match-play. Journal of Human Movement Studies, 2, 87-97.

Sanderson, F. H., \& Way, K. I. (1977). The development of objective methods of game analysis in squash rackets. British Journal of Sports Medicine, 11, 188-188.

Sarro, K. J., Misuta, M. S., Burkett, B., Malone, L. A., \& Barros, R. M. L. (2010). Tracking of wheelchair rugby players in the 2008 demolition derby final. Journal of Sports Sciences, 28, 193-200.

Sathyan, T., Humphrey, D., \& Hedley, M. (2011). WASP: A system and algorithms for accurate radio localization using low-cost hardware. Systems, Man, and Cybernetics, Part C: Applications and Reviews, IEEE Transactions on, 41, 211-222. 
Sathyan, T., Shuttleworth, R., Hedley, M., \& Davids, K. (2012). Validity and reliability of a radio positioning system for tracking athletes in indoor and outdoor team sports. Behavior Research Methods, 44, 1108-1114.

Schutz, Y., \& Chambaz, A. (1997). Could a satellite-based navigation system (GPS) be used to assess the physical activity of individuals on earth? European Journal of Clinical Nutrition, 51, 338-339.

Siegle, M., Stevens, T., \& Lames, M. (2013). Design of an accuracy study for position detection in football. Journal of Sports Sciences, 31, 166-172.

Sindall, P., Lenton, J. P., Tolfrey, K., Cooper, R. A., Oyster, M., \& Goosey-Tolfrey, V. L. (2013a). Wheelchair tennis match-play demands: Effect of player rank and result. International Journal of Sports Physiology and Performance, 8, 28-37.

Sindall, P., Whytock, K., Lenton, P., Tolfrey, K., Oyster, M., Cooper, R., \& Goosey-Tolfrey, V. (2013b). Criterion validity and accuracy of global positioning satellite and data logging devices for wheelchair tennis court movement. The Journal of Spinal Cord Medicine, 36, 383-393.

Sporner, M. L., Grindle, G. G., Kelleher, A., Teodorski, E. E., Cooper, R., \& Cooper, R. A. (2009). Quantification of activity during wheelchair basketball and rugby at the national veteran's wheelchair games: A pilot study. Prosthetics and Orthotics International, 33, 210-217.

Stelzer, A., Pourvoyeur, K., \& Fischer, A. (2004). Concept and application of LPM-a novel 3-D local position measurement system. Microwave Theory and Techniques, IEEE Transactions on, 52, 2664-2669.

Vanlandewijck, Y., Spaepen, A., Lysens, R. (1994) Wheelchair propulsion efficiency: movement pattern adaptations to speed changes. Medicine \& Science in Sports \& Exercise, 26, 1373-1381.

Vanlandewijck, Y., Theisen, D., \& Daly, D. (2001). Wheelchair propulsion biomechanics. Sports Medicine, 31, 339-367.

Varley, M. C., Fairweather, I. H., \& Aughey1, 2, R. J. (2012). Validity and reliability of GPS for measuring instantaneous velocity during acceleration, deceleration, and constant motion. Journal of Sports Sciences, 30, 121-127.

Venter, R., Opperman, E., \& Opperman, S. (2011) The use of global position system (GPS) tracking devices to assess movement demands and impacts in under-19 rugby union match play. African Journal for Physical, Health Education, Recreation and Dance, 17, 1-8. 


\section{$1 \quad$ Figure Legends}

2 Figure 1: The location of the nine tags fixed to the wheelchair during dynamic tests. Inset is 3 the sampling frequency for each tag with regards to its location.

4 Figure 2: The 'figure of eight' drill used to assess distance during incremental fixed speeds.

5 The solid middle horizontal line represents the location of the timing gates and the start/finish 6 of the drill $(\mathrm{a}=8 \mathrm{~m}, \mathrm{~b}=12.25 \mathrm{~m}$; lap $=81 \mathrm{~m}$; total distance $=405 \mathrm{~m})$.

$7 \quad$ Figure 3: Plot of mean static error for each sampling frequency during pre and post session.

8 Error bars represent standard deviation.

$9 \quad$ Figure 4: Plot of mean error (bias) for each frequency during maximal sprint tests $\left(\mathrm{m} \cdot \mathrm{s}^{-1}\right)$. 10 Error bars represent 95\% LOA. * represents a significant difference between sampling 11 frequency and criterion.

12 Figure 5: Plot of mean error (bias) for distance $(\mathrm{m})$ and mean speed $\left(\mathrm{m} \cdot \mathrm{s}^{-1}\right)$ during the multi13 directional test. Error bars represent 95\% LOA. 
Table 1. Distance and mean speed values during movement at incremental fixed speeds (test ii)

\begin{tabular}{|c|c|c|c|c|c|c|c|c|c|c|c|c|c|c|}
\hline \multirow[b]{2}{*}{ Speed } & \multicolumn{7}{|c|}{ Distance $(\mathrm{m})$} & \multicolumn{7}{|c|}{ Mean Speed $\left(\mathrm{m} \cdot \mathrm{s}^{-1}\right)$} \\
\hline & Criterion & Low Freq & TEE & Med Freq & TEE & High Freq & TEE & Criterion & Low Freq & TEE & Med Freq & TEE & High Freq & TEE \\
\hline Low & 395 & 404 (399-409) & 1.96 & $402(399-406)$ & 1.85 & $400(398-404)$ & 2.11 & 1.14 & $1.17(1.15-1.19)$ & 0.01 & $1.16(1.14-1.18)$ & 0.01 & $1.15(1.14-1.17)$ & 0.01 \\
\hline Med & 394 & $398(397-399)$ & 1.03 & $398(396-400)$ & 1.04 & $396(394-398)$ & 1.09 & 1.55 & $1.57(1.52-1.60)$ & 0.01 & $1.56(1.51-1.60)$ & 0.01 & $1.56(1.51-1.59)$ & 0.01 \\
\hline High & 394 & $397(394-400)$ & 1.06 & $397(395-401)$ & 1.00 & $396(394-399)$ & 0.98 & 2.01 & $2.03(1.97-2.07)$ & 0.01 & $2.02(1.96-2.06)$ & 0.01 & $2.01(1.95-2.05)$ & 0.01 \\
\hline
\end{tabular}

Mean values (95\% confidence limits)

TEE expressed as raw units 
